\title{
Optimización del proceso de Fito remediación con Festuca Dolichophylla y tiosulafto de amonio para mejorar la eficacia de la absorción del mercurio de suelos contaminados con mercurio por la minería del distrito de Ananea Puno-Perú
}

\author{
Optimization of the process of Phytoremediation whit Festuca Dolichophylla \\ and ammonium thiosulphate to improve the effectiveness of mercury \\ absorption from soils contaminated whit mercury by mining in the district of \\ Ananea Puno-Peru
}

\author{
Siles Nilo Arce Sancho ${ }^{1}$, Julia Marilú Calderón Celis ${ }^{2}$, Yesith Jarecca Cusacani ${ }^{3}$
}

Recibido: 26/02/2021 - Aprobado: 19/09/2021 - Publicado: 23/12/2021

\begin{abstract}
RESUMEN
Un pasivo ambiental en el Perú se encuentra en el distrito de Ananea (Puno), fue originado por actividades mineras informales desde hace 90 años. Se realizó fitorremediación con Festuca Dolichophylla planta nativa del lugar añadiendo tiosulfato de amonio como acelerador químico. Las muestras de suelos se extrajeron de las áreas impactadas con mercurio, en la investigación se utilizó el diseño factorial que tuvo como variables respuesta: la concentración de mercurio total ( $\mathrm{HgT}$ ) en suelo y en los tejidos vegetales, y como factores el nivel de tiosulfato de amonio en el suelo y el tiempo de crecimiento de la planta. Los suelos del distrito de Ananea-Puno tienen $\mathrm{pH}$ neutro, el mercurio elemental constituye el $56 \%$ de la cantidad total de mercurio en el suelo. Las concentraciones de THg en el suelo oscilan entre 44 y $53 \mathrm{mg} / \mathrm{kg}$, que es casi 2 veces mayor que el límite superior para el contenido de mercurio $(24 \mathrm{mg} / \mathrm{kg}$ ) en suelos extractivos en Perú. El BAF (Factor de bioacumulación) que se obtuvo fue de 0.38 en las hojas, para $2 \mathrm{~g}$ de tiosulfato de amonio por $\mathrm{Kg}$ de suelo, lo que indica que la Festuca Dolichopylla es una planta no hiperacumuladora. El contenido de THg se redujo en el suelo en relación con el suelo inicial, en $43.67 \mathrm{mg} / \mathrm{kg}$, obteniéndose una eficacia de hasta $82 \%$.
\end{abstract}

Palabras claves: Mercurio; fitorremediación; suelos contaminados; Festuca Dolichophylla; tiosulfato de amonio.

\begin{abstract}
An environmental liability in Peru is located in Puno, in the district of Ananea, originated by informal mining activities 90 years ago. Phytoremediation was carried out with Festuca Dolichophylla, a plant native to the area, with the assistance of ammonium thiosulfate as an accelerator. Soil samples were extracted from the areas impacted with mercury. The research used a factorial design with the following response variables: the concentration of total mercury $(\mathrm{HgT})$ in soil and plant tissues, and as factors the level of ammonium thiosulfate in the soil and the time of plant growth. The soils of the Ananea-Puno district have neutral pH, elemental mercury constitutes $56 \%$ of the total amount of mercury in the soil. THg concentrations in the soil range from 44 to $53 \mathrm{mg} / \mathrm{kg}$, which is almost 2 times higher than the upper limit for mercury content $(24 \mathrm{mg} / \mathrm{kg})$ in extractive soils in Peru. The BAF (Bioaccumulation Factor) obtained was 0.38 in leaves, for $2 \mathrm{~g}$ of ammonium thiosulfate per $\mathrm{kg}$ of soil, indicating that Festuca Dolichopylla is a non-hyperaccumulator plant. The THg content was reduced in the soil in relation to the initial soil, by $43.67 \mathrm{mg} / \mathrm{kg}$, obtaining an efficiency of up to $82 \%$.
\end{abstract}

Keywords: Mercury; phytoremediation; contaminated soils; Festuca Dolichophylla; ammonium thiosulphate.

\footnotetext{
1 Doe Run Perú SRL. Universidad Nacional Mayor de San Marcos, Unidad de Posgrado, Lima, Perú. Egresado doctorado Ciencias Ambientales Autor para correspondencia: siles.arce@unmsm.edu.pe - ORCID: https://orcid.org/0000-0002-7987-5074

2 Universidad Nacional Mayor de San Marcos, Lima, Perú. Docente Principal, Investigadora, Asesora de Tesis de Pregrado y Posgrado. E-mail: jcalderond2@unmsm.edu.pe / calderonmarilu@gmail.com ORCID: https://orcid.org/0000-0002-1374-9307

3 Universidad Nacional de Juliaca, Perú

E-mail: ym.jareccac@unaj.edu.pe - ORCID: https://orcid.org/0000-0002-0696-9184
} 


\section{INTRODUCCIÓN}

Uno de los pasivos ambientales importantes presentes en el Perú son los que se encuentran en el departamento de Puno, provincia de Putina, en el distrito de Ananea, las actividades mineras artesanales informales existentes en dicho distrito, se han venido incrementándose desde los años 90 intensificándose en los últimos 8 años, contaminado el área de influencia directa como la cuenca del Ramis con metales pesados como es el mercurio (Chávez Rodríguez, 2014).

En el departamento de Puno, alrededor de 15 toneladas de mercurio líquido se pierden cada año. y estaría aumentando en los últimos años, a razón de un 10\% anual esto por el incremento de producción de oro y falta de formalización de la minería informal, Figura 1 (Macera Poli, 2018).

El uso sin control y de forma ineficiente del mercurio para amalgamar el oro, origina que cantidades considerables de esta sustancia se echen a perder para ser vertidos al medioambiente en forma líquida cuando se hace la amalgamación o se emane en forma gaseosa cuando se hace el refogado. La pérdida de mercurio es aproximadamente de diez gramos por tonelada de mineral tratado, siempre y cuando se tengan los cuidados necesarios para el trabajo de amalgamación, de otra forma las pérdidas son mayores.

Ante las alarmantes consecuencias de los metales pesados como el mercurio, en la salud humana y en los ecosistemas es que es necesario buscar metodologías que permitan reducir el contenido de estos metales pesados, en los lugares donde se encuentra acumulados, como el suelo, cuerpos de agua y aire. En el caso de Ananea se observó que hay una acumulación de $\mathrm{Hg}$ por encima del ECA suelo según normatividad peruana que es de $24 \mathrm{mg} / \mathrm{kg}$ para un suelo comercial, industrial o extractivo y $6.6 \mathrm{mg} / \mathrm{kg}$ para suelo agrícola y residencial (SINIA, 2015).

Los tratamientos físicos-químicos convencionales resultan muy caros y poco convenientes para ser aplicados en grandes extensiones, la fitorremediación ha probado ser una buena técnica para recuperar estos suelos, siendo clave la selección de especies vegetales apropiadas para realizar la fitorremediación y que tengan capacidad para absorber, metabolizar, acumular, estabilizar o volatilizar metales pesados como el mercurio (Dorronsoro \& García, 2005).

La tecnología de fitorremediación tiene algunas desventajas, no se puede aplicar a suelos muy contaminados, el $\mathrm{Hg}$ interfiere en el proceso metabólico de las plantas y obstaculiza su crecimiento, y el tiempo de remediación es largo. Se suele utilizar la asistencia química para mejorar la eficacia de la fitorremediación (Liu et al., 2020).

En el estudio de plantas que se desarrollan en el área contaminada de Ananea como es el caso del Stipa Ichu, dieron resultados en la absorción de plomo y mercurio, absorbiendo $310,45 \mathrm{mg} / \mathrm{kg}$ de planta. Apoyando la hipótesis de que las plantas nativas tienen más probabilidades de extraer metales pesados en sus propios espacios naturales (Ortiz \& Aranibar, 2015). En el presente estudio se eligió otra planta nativa de similares características que es la Festuca dolichophylla.

La presente investigación tiene como objetivo determinar la eficacia de fitorremediación de la Festuca Dolichophylla en suelos que están contaminados con mercurio por actividades mineras del distrito de Ananea Puno-Perú bajo condiciones semi-controladas del uso de tiosulfato de amonio como acelerador químico.

\section{METODOS}

En presente trabajo de investigación se desarrolló bajo condiciones controladas empleando un diseño factorial (Arnau, J., \& Bono, 1997), que tuvo como variables dependientes (respuesta) la concentración de mercurio total $(\mathrm{HgT})$ en en los tejidos vegetales y en el suelo, y como factores: el tiempo de crecimiento de la planta (4

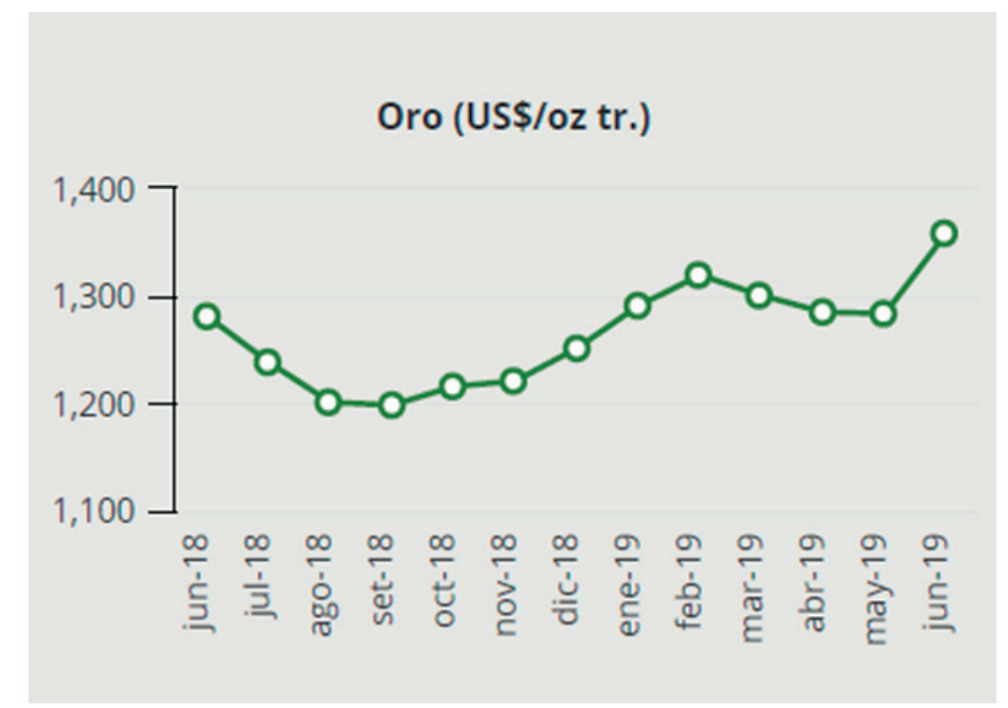

Figura 1. Producción de oro en Puno. Dirección de Promoción Minera del Ministerio de Energía y Minas (MINEM, 2020) 
niveles: mes 0 , mes 3 , mes 6 , y mes 9) y contaminación del suelo (4 niveles: suelo contaminado sin ninguna adición, suelo contaminado con tiosulfato de amonio con una concentración de $1 \mathrm{~g} / \mathrm{kg}$, suelo contaminado con tiosulfato de amonio con una concentración de $2 \mathrm{~g} / \mathrm{kg}$ y suelo control).

El tratamiento para el suelo control consistió en las siembra de las plantas en un suelo con una muy baja concentración de $\mathrm{Hg}$, (tendencia a 0 ) con el fin de diferenciar las características fitotóxicas con las plantas expuestas a altas concentraciones de $\mathrm{Hg}$, ya que las plantas que fueron sembradas en el suelo control no dan altas tasas de acumulación de mercurio en la planta. Ni dan altas tasas de remoción del metal del suelo (Teng et al., 2020).

La cantidad de plantas sembradas (repeticiones) por combinación de niveles de factores fue de 3 , razón por la cual se sembraron 12 plantas de Festuca Dolychopylla en cada uno de los suelos en forma aleatoria. Las muestras de las plantas se tomaron cada tres meses hasta cumplir 9 meses de crecimiento y luego fueron enviadas al laboratorio para la determinación del mercurio total (HgT) Tambien, se determinó la concentración de mercurio total (HgT) y mercurio biodisponible en los suelos, a los 0,3, 6 y 9 meses para determinar el porcentaje de remoción de mercurio del suelo (Wang et al., 2018).

De acuerdo con el tamaño de muestra se necesitó extraer muestras de suelo de áreas impactadas directamente por actividades mineras del distrito de Ananea. Tabla 1

Seleccionados los lugares de muestreo, la toma de muestras superficiales se aplicó el protocolo de muestreo de suelos del MINAM (2014).

Según el diseño experimental se necesitó 1 kilo por unidad de análisis. Pero se efectuó por triplicado (3 muestras por unidad de análisis) por ser una configuración cuadrática de los diseños factoriales. (Arnau \& Bono, 2017). Por lo que se trasplantaron 36 plantas de Festuca Doichopylla de forma individual en $1 \mathrm{~kg}$ de suelo/u (unidad experimental) contaminados con mercurio de acuerdo con el diseño experimental y se llevaron a macetas, estas macetas fueron ubicadas en un área experimental al aire libre, a condiciones normales, cuidando de los incrementos bruscos de luz y temperatura. A las plantas se regaron con agua de caño dos veces al día a tres cuartos de la capacidad de las macetas y se hizo un monitoreo permanente de las características morfológicas propias de las plantas con el fin de identificar algunos efectos tóxicos que pudieran ser causados por los suelos contaminados con $\mathrm{Hg}$ en las plantas (Geng et al., 2019).

Las unidades de análisis estuvieron dadas por la combinación de los 2 factores (contaminación de suelos, y tiempo de crecimiento de las plantas) el primero con 4 niveles y el segundo con 4 niveles entonces se tuvo un diseño factorial 4 x 4 . Los cuales se detallan en la tabla 2 .

Para la extracción de plantas Festuca Dolichopylla, se seleccionó un área limpia que no estuvo expuesta a contaminación con mercurio, excavando desde el tallo principal a un radio de $20 \mathrm{~cm}$ de distancia, esto para cada una de las plantas, con el objetivo de proteger las raíces.

Cuando ya se recolectaron las plantas se hicieron grupos de a dos y se colocaron en recipientes de plástico que se llenaron con tierra procedente de los puntos de extracción con el fin de cubrir las raíces y cuidar de que estén expuestas a la luz solar. (Lui, et al., 2020)

Se colectaron 3 muestras de planta del punto seleccionado de muestreo teniendo en cuenta siempre las condiciones meteorológicas del campo. Para ello se escogieron plantas de mediano tamaño, mediana edad y que tengan buen crecimiento radicular. Se realizó el traslado de estas plantas siempre teniendo cuidado de que las raíces no se expongan a la luz solar a fin de que no se marchiten (Lomonte et al., 2010).

Inicialmente se trasplantaron 36 plántulas de Festuca Dolychopilla jóvenes y de similares características (16 hojas) individualmente en $1 \mathrm{~kg}$ de suelo que constituye una unidad experimental libres de mercurio y se trasladaron a macetas, una vez que estas plantas estuvieron estables fueron trasplantados a otras macetas que estuvieron ya

Tabla 1. Áreas de extracción de suelos contaminados con mercurio

\begin{tabular}{cccc}
\hline Área de extracción & A & B & C \\
\hline Coordenadas & $14^{\circ} 40^{\prime} 01.0^{\prime \prime}$ S; $69^{\circ} 28^{\prime} 03.3^{\prime \prime} \mathrm{W}$. & $14^{\circ} 38^{\prime} 29.0^{\prime \prime} \mathrm{S} ; 69^{\circ} 27^{\prime} 31.41^{\prime \prime} \mathrm{W}$. & $14^{\circ} 40^{\prime} 33.0^{\prime \prime S} ; 69^{\circ} 32^{\prime} 42.3^{\prime \prime} \mathrm{W}$. \\
Nombre de la zona & Pampa Blanca. & La Rinconada & Ananea \\
Altitud & 4841 m.s.n.m. & 4980 m.s.n.m. & 4680 m.s.n.m. \\
Área aproximada & 100 Ha & 5 Ha. & 20 Ha. \\
\hline
\end{tabular}

Tabla 2. Combinaciones de factores (Contaminación suelo Vs Tiempo de crecimiento de la planta)

\begin{tabular}{lcccc}
\hline & Suelo Control & Suelo contaminado & $\begin{array}{c}\text { Suelo contaminado con tiosulfato de amonio } \\
\text { C1 }\end{array}$ & $\begin{array}{c}\text { Suelo contaminado con tiosulfato de amonio } \\
\text { C2 }\end{array}$ \\
\hline 0 meses & $\mathrm{U}(0,1)$ & $\mathrm{U}(0,2)$ & $\mathrm{U}(0,3)$ & $\mathrm{U}(0,4)$ \\
3 meses & $\mathrm{U}(1,1)$ & $\mathrm{U}(1,2)$ & $\mathrm{U}(1,3)$ & $\mathrm{U}(1,4)$ \\
6 meses & $\mathrm{U}(2,1)$ & $\mathrm{U}(2,2)$ & $\mathrm{U}(2,3)$ & $\mathrm{U}(2,4)$ \\
9 meses & $\mathrm{U}(3,1)$ & $\mathrm{U}(3,2)$ & $\mathrm{U}(3,3)$ & $\mathrm{U}(3,4)$ \\
\hline
\end{tabular}


con suelo contaminado de acuerdo a tabla del diseño experimental, estas macetas fueron ubicadas en un área experimental al aire libre dentro de las instalaciones de la Universidad Nacional de Juliaca, a condiciones normales climáticas propias del área, sin cambios bruscos de luz y temperatura.

Las plantas en su totalidad se dispusieron en un diseño de bloques al azar con una temperatura diurna promedio de 15 a $20{ }^{\circ} \mathrm{C}$ y una humedad de 40 a $60 \%$. Se les regó diariamente con agua del grifo a las macetas para mantener un nivel de humedad justo por debajo de la capacidad de agua, para evitar la liberación de lixiviados de las macetas y se realizó un monitoreo de las características morfológicas propias de la planta como son: la longitud de la planta, número de hojas, con la finalidad de identificar algunos posibles efectos tóxicos causados por los suelos contaminados con $\mathrm{Hg}$ sobre las plantas de Festuca Dolychopilla.

Cinco días antes de la cosecha (9 meses) se añadió la solución de $\left(\mathrm{NH}_{4}\right) \cdot 2 \mathrm{~S}_{2} \mathrm{O}_{3}$ de 1 y $2 \mathrm{~g}$ de tiosulfato por $\mathrm{Kg}$ de suelo a las macetas según cuadro de diseño, mientras que en lugar del reactivo se añadió agua destilada a las macetas del blanco y a las macetas control (Wang et al., 2018). Todas las macetas recibieron un volumen de tratamiento de 200 $\mathrm{ml}$ de solución. Cinco días después de la adición de $\left(\mathrm{NH}_{4}\right)$ $2 \mathrm{~S}_{2} \mathrm{O}_{3}$, todas las plantas se cosecharon y se dividieron en hojas, tallos y raíces, que se lavaron minuciosamente con agua del grifo seguido de agua destilada y luego se secaron al aire (Wang et al., 2011).

Se registró el peso del tejido vegetal (peso seco). También se recolectó las muestras de suelo en el momento de la cosecha. Todas las muestras de suelo se secaron al aire, se trituraron y se tamizaron hasta una malla 150 .Se midieron las propiedades de la muestra de suelo: como $\mathrm{pH}$, textura y materia orgánica (MO).

El mercurio elemental $(\mathrm{Hg} 0)$ de las muestras de suelo se evaluó según el método de: García-Sánchez et al. (2009). Se colocaron submuestras de suelo (1 g) en crisoles de porcelana y se calentaron a $180{ }^{\circ} \mathrm{C}$ durante 24 $\mathrm{h}$ en un horno. Posteriormente, estas muestras calentadas se analizaron para determinar el contenido de mercurio restante. Las concentraciones de $\mathrm{Hg}_{0}$ se derivaron de la diferencia entre el contenido total de mercurio analizado antes y después del proceso de calentamiento. Para el análisis de $\mathrm{THg}$, las muestras de suelo se digirieran en un baño de agua $\left(95^{\circ} \mathrm{C}\right)$ usando una mezcla fresca de $\mathrm{HCl}$ concentrado y $\mathrm{HNO}_{3}(3: 1, \mathrm{v} / \mathrm{v})$. Las muestras de plantas se digirieran con $\mathrm{HNO}_{3}$ concentrado (Marrugo-Negrete et al., 2020).

El THg de las muestras de suelo recolectadas de las macetas y suelos se midieron mediante espectrofotometría de absorción atómica de vapor frío en la UNA Puno.

\section{RESULTADOS}

\subsection{Propiedades fisicoquímicas del suelo}

Las propiedades fisicoquímicas de suelos recolectados del distrito minero de Ananea se resumen en la Tabla 3.

Los suelos tienen un $\mathrm{pH}$ neutro (entre 6,88 y 7,04. La forma elemental de mercurio aproximadamente constituyó el $56 \%$ de la cantidad total de mercurio en el suelo. Las concentraciones de $\mathrm{THg}$ en el suelo oscilaron entre $44 \mathrm{y}$ $53 \mathrm{mg} / \mathrm{kg}$, que es casi 2 veces mayor que el límite superior máximo para el contenido de mercurio $(24 \mathrm{mg} / \mathrm{kg})$ en suelos extractivos en Perú (SINIA, 2015) y, por tanto, el suelo puede considerarse contaminado. Las concentraciones de $\mathrm{THg}$ en el suelo de base varían de 0,12 a $0,14 \mathrm{mg} / \mathrm{kg}$.

\subsection{Acumulación vegetal inducida}

La concentración del mercurio total $(\mathrm{THg})$ en raíces, tallos y hojas de la Festuca Dolichopylla después del tratamiento con tiosulfato se resume en la figura 2. La concentración de $\mathrm{THg}$ se incrementó $600 \%, 700 \%$ y $300 \%$ en raíces, tallos y hojas de Festuca Dolichopylla. para las macetas tratadas con $2 \mathrm{~g} / \mathrm{kg}$ de tiosulfato, respectivamente, en relación con el no tratado. Ver Tabla 4:

Graficando los resultados de la tabla 4 se puede observar que hay una mayor acumulación en las hojas, seguido por la raíz y el tallo, la mayor acumulación se da a mayor concentración de tiosulfato. Figura 2.

\subsection{Determinación de dependencia de las variables en estudio}

De acuerdo con la tabla 2 se ha efectuado la corrida experimental, y los resultados se muestran en la tabla 5.

Usando el paquete estadístico $\mathrm{R}$ se ha calculado los coeficientes de correlación multivariados a un nivel de

Tabla 3. Propiedades fisicoquímicas del suelo

\begin{tabular}{|c|c|c|c|c|}
\hline Parámetros del suelo & Pampa Blanca. & La Rinconada & Ananea & Compósito \\
\hline $\mathrm{pH}$ & 6.88 & 6.91 & 7.02 & 6.92 \\
\hline $\mathrm{CE}\left(\mu \mathrm{S} \mathrm{cm}{ }^{-1}\right)$ & 304 & 310 & 319 & 311 \\
\hline $\mathrm{OM}\left(\mathrm{g} \mathrm{kg}^{-1}\right)$ & 49.4 & 43.6 & 52.3 & 48.8 \\
\hline Densidad de suelo $\left(\mathrm{g} / \mathrm{cm}^{-3}\right)$ & 1.17 & 1.21 & 1.19 & 1.19 \\
\hline Distribución de tamaño de partícula (\%) & $\begin{array}{l}\text { Arena } 15.36 \\
\text { Limo } 55.96 \\
\text { Arcilla } 28.67\end{array}$ & $\begin{array}{l}\text { Arena } 13.86 \\
\text { Limo } 52.48 \\
\text { Arcilla } 33.66\end{array}$ & $\begin{array}{l}\text { Arena } 12.32 \\
\text { Limo } 51.00 \\
\text { Arcilla } 36.68\end{array}$ & $\begin{array}{l}\text { Arena } 15.36 \\
\text { Limo } 55.96 \\
\text { Arcilla } 28.67\end{array}$ \\
\hline Mercurio total (mg Kg-1) & 51.13 & 53.14 & 44.00 & 49.4 \\
\hline Elemento $\mathrm{Hg}\left(\mathrm{mg} \mathrm{Kg}^{-1}\right)$ & 27 & 35 & 21 & 28 \\
\hline
\end{tabular}


Tabla 4. Peso seco y masa total de mercurio de la hoja tallo y raíz de una sola planta (promedio) $n=3$

\begin{tabular}{|c|c|c|c|c|c|}
\hline & & Suelo Control & Suelo contaminado & $\begin{array}{l}\text { Suelo contaminado con tiosulfato } \\
\text { de amonio C1 }\end{array}$ & $\begin{array}{l}\text { Suelo contaminado con tiosulfato } \\
\text { de amonio C2 }\end{array}$ \\
\hline \multirow[t]{2}{*}{ Hoja } & Peso seco (g) & $\begin{array}{l}\mu=3.58 \\
\delta=0.78\end{array}$ & $\begin{array}{l}\mu=3.36 \\
\delta=0.82\end{array}$ & $\begin{array}{l}\mu=4.68 \\
\delta=0.67\end{array}$ & $\begin{array}{l}\mu=4.76 \\
\delta=0.60\end{array}$ \\
\hline & Masa THg $(\mu \mathrm{g})$ & $\begin{array}{l}\mu=1.13 \\
\delta=0.55\end{array}$ & $\begin{array}{l}\mu=8.43 \\
\delta=1.55\end{array}$ & $\begin{aligned} \mu & =19.67 \\
\delta & =1.62\end{aligned}$ & $\begin{aligned} \mu & =25.90 \\
\delta & =1.34\end{aligned}$ \\
\hline \multirow[t]{2}{*}{ tallo } & Peso seco (g) & $\begin{array}{l}\mu=4.29 \\
\delta=0.77\end{array}$ & $\begin{array}{l}\mu=5.09 \\
\delta=0.87\end{array}$ & $\begin{array}{l}\mu=3.70 \\
\delta=0.72\end{array}$ & $\begin{array}{l}\mu=5.70 \\
\delta=0.99\end{array}$ \\
\hline & Masa THg $(\mu \mathrm{g})$ & $\begin{array}{l}\mu=0.58 \\
\delta=0.06\end{array}$ & $\begin{array}{l}\mu=0.70 \\
\delta=0.12\end{array}$ & $\begin{array}{l}\mu=3.06 \\
\delta=0.76\end{array}$ & $\begin{array}{l}\mu=5.06 \\
\delta=0.36\end{array}$ \\
\hline \multirow[t]{2}{*}{ Raiz } & Peso seco (g) & $\begin{array}{l}\mu=0.98 \\
\delta=0.13\end{array}$ & $\begin{array}{l}\mu=1.57 \\
\delta=0.08\end{array}$ & $\begin{array}{l}\mu=0.51 \\
\delta=0.22\end{array}$ & $\begin{array}{l}\mu=0.81 \\
\delta=0.32\end{array}$ \\
\hline & Masa THg $(\mu \mathrm{g})$ & $\begin{array}{l}\mu=0.40 \\
\delta=0.08\end{array}$ & $\begin{array}{l}\mu=1.40 \\
\delta=0.09\end{array}$ & $\begin{aligned} \mu & =4.54 \\
\delta & =2.61\end{aligned}$ & $\begin{array}{l}\mu=8.54 \\
\delta=1.21\end{array}$ \\
\hline
\end{tabular}

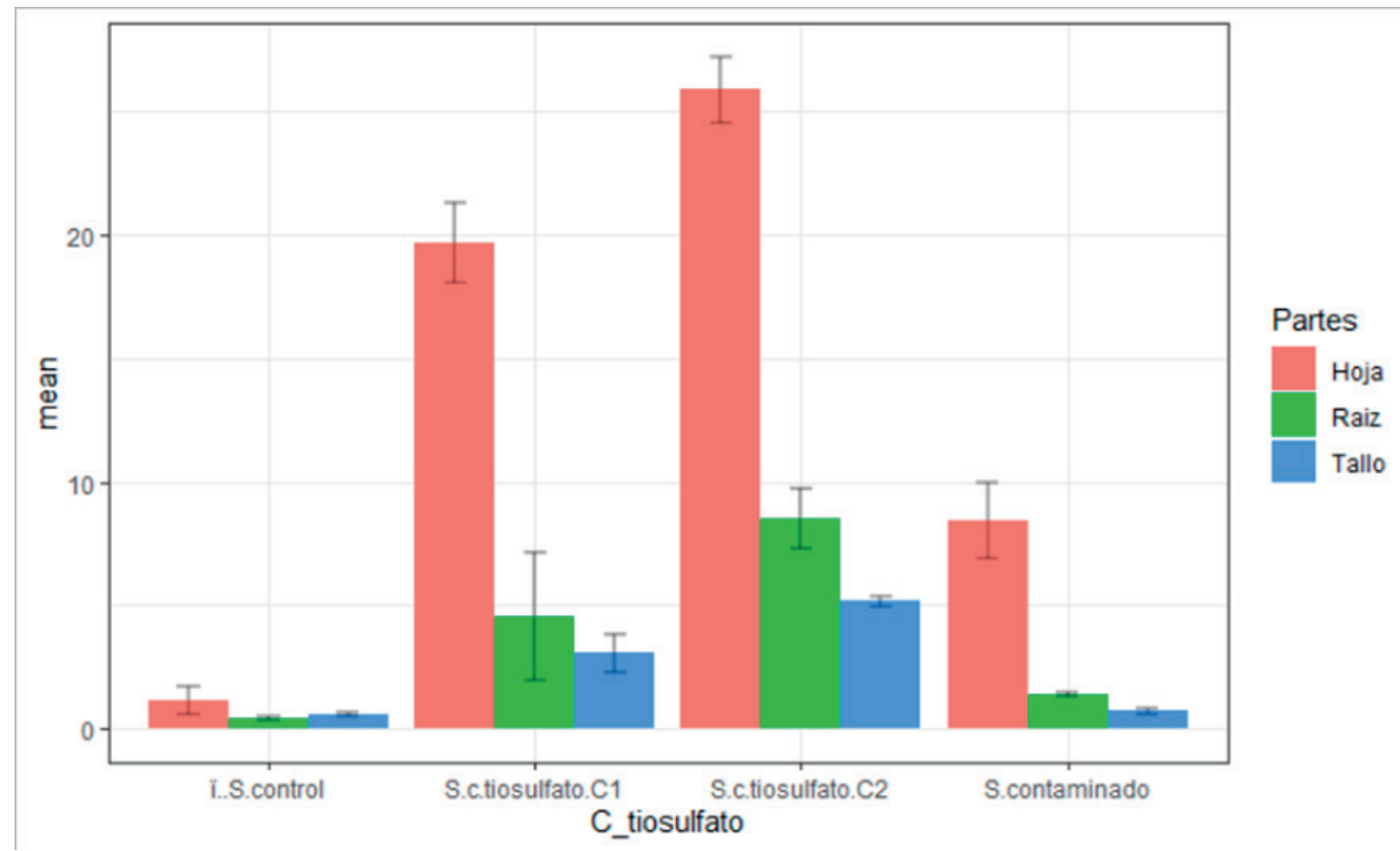

Figura 2. Concentración de THg en raíz, tallo y hoja en macetas control y tratadas con tiosulfato de amonio. Las barras denotan la desviación estándar de la media de tres repeticiones.

Tabla 5. Resultados de contaminante suelo para la Festuca Dolychopilla

\begin{tabular}{|c|c|c|c|c|}
\hline Tiempo & $\begin{array}{l}\text { Suelo Control } \\
\mathrm{mg} / \mathrm{Kg}\end{array}$ & $\begin{array}{l}\text { Suelo contaminado } \\
\mathrm{C}_{0} \mathrm{mg} / \mathrm{Kg}\end{array}$ & $\begin{array}{l}\text { Suelo contaminado con tiosulfato } \\
\text { de amonio } C_{1}\end{array}$ & $\begin{array}{l}\text { Suelo contaminado con tiosulfato } \\
\text { de amonio } C_{2}\end{array}$ \\
\hline 0 meses & $\begin{array}{l}\mu=0.19 \\
\delta=0.0003\end{array}$ & $\begin{array}{l}\mu=49.4 \\
\delta=0.34\end{array}$ & $\begin{array}{l}\mu=49.3 \\
\delta=0.33\end{array}$ & $\begin{array}{l}\mu=49.0 \\
\delta=0.31\end{array}$ \\
\hline 3 meses & $\begin{array}{l}\mu=0.17 \\
\delta=0.0005\end{array}$ & $\begin{aligned} \mu & =38.3 \\
\delta & =0.4\end{aligned}$ & $\begin{array}{l}\mu=26.3 \\
\delta=0.38\end{array}$ & $\begin{array}{l}\mu=12.4 \\
\delta=0.46\end{array}$ \\
\hline 6 meses & $\begin{array}{l}\mu=0.13 \\
\delta=0.0005\end{array}$ & $\begin{aligned} \mu & =23.4 \\
\delta & =1.3\end{aligned}$ & $\begin{array}{l}\mu=10.4 \\
\delta=0.95\end{array}$ & $\begin{array}{l}\mu=8.3 \\
\delta=0.34\end{array}$ \\
\hline 9 meses & $\begin{array}{l}\mu=0.12 \\
\delta=0.0004\end{array}$ & $\begin{array}{l}\mu=15.4 \\
\delta=1.11\end{array}$ & $\begin{array}{l}\mu=8.2 \\
\delta=1.01\end{array}$ & $\begin{array}{l}\mu=5.3 \\
\delta=0.89\end{array}$ \\
\hline
\end{tabular}


significancia de $95 \%$. Figura 3 y se ha calculado el valor estadístico F crítico que resulta 0.0445 que es $<$ a 0.05 dada a esta comparación, se determina que si hay dependencias de las variables en estudio (tiempo y concentración con tiosulfato respecto al contenido de $\mathrm{Hg}$ en el suelo).

\subsection{Determinación del factor de bioacumulación}

El factor de bioacumulación (BAF) del mercurio por Festuca Dolychopylla se usa la siguiente ecuación.

Factor bioacumulación $($ BAFTotal $)=$ Concentración $\underline{\text { THg brote de la planta }}$

THg en el suelo

Concentración de

El BAF que se muestra en la tabla 6, se calculó en base a los datos de la tabla 4 y tabla 5 , donde se observa que la concentración total de mercurio es mayor en la hoja, raíz y tallo respectivamente para plantas tratadas con tiosulfato en relación con las no tratadas. Esto respalda la observación de que el tiosulfato puede aumentar la absorción de mercurio por Festuca Dolychopylla.

El BAF se obtiene utilizando la concentración extraída de mercurio en el suelo al final del experimento, se observa que el BAF es mayor para el suelo tratado con tiosulfato con mayor concentración $(2 \mathrm{~g} / \mathrm{kg})$, sin embargo, dado que es un valor menor a la unidad se determina que la Festuca Dolichopylla no es hiperacumuladora de mercurio pero dado su potencial fitoextractor puede ser usado en forma práctica.

\section{DISCUSIÓN}

$\mathrm{Si}$ se quiere comparar los resultados de la presente investigación con otras investigaciones se tiene en la Tabla 7.

En la tabla 7 se observa que la Festuca Dolichopylla absorbe hasta $16.87 \mathrm{mg} / \mathrm{kg}$ en las hojas usando tiosulfato el cual es superior al de otras plantas a excepción de la Brassica juncea, lo que nos demuestra que si puede ser considerado como un buen fitorremediador para el mercurio.

Suponiendo una producción promedio de biomasa de brotes (peso seco) para Festuca Dolichopylla de 11270 kg/ ha (resultado extrapolado del experimento de macetas), y la concentración promedio de mercurio de brotes (hoja + tallo) obtenida en este estudio $(6.55 \mathrm{mg} / \mathrm{kg})$, el rendimiento de la fitoextracción de mercurio asistida por tiosulfato es de $73 \mathrm{~g} / \mathrm{ha}$. Dado que la concentración de $\mathrm{THg}$ en el suelo es de $50 \mathrm{mg} / \mathrm{kg}$, la fitoextracción, como se demuestra en esta investigación, es una tecnología viable para disminuir la concentración de $\mathrm{THg}$ del suelo de Ananea a un nivel seguro. Este punto debe considerarse en la revegetación de desechos mineros contaminados con $\mathrm{Hg}$.

Teniendo en cuenta nuestros resultados, creemos que la fitorremediación de mercurio con la Festuca Dolichopylla asistida por tiosulfato podría potencialmente usarse para manejar el nivel de contaminación de mercurio

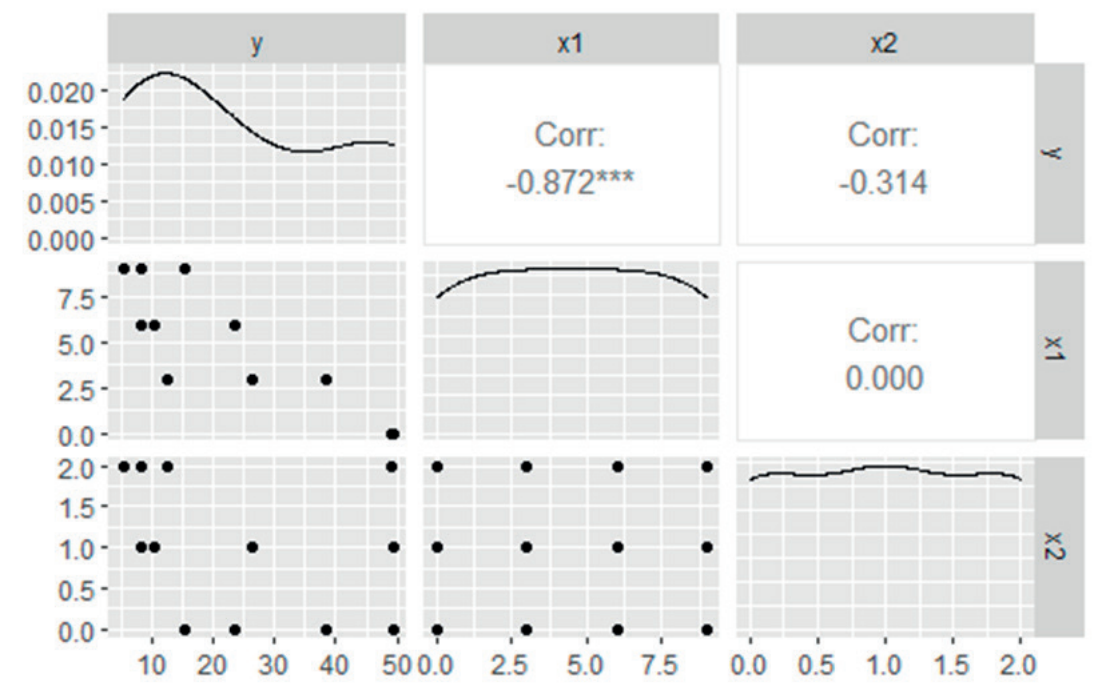

Figura 3. Análisis de correlación de variable múltiple.

Tabla 6. BAF de la Festuca Dolichopylla

\begin{tabular}{|c|c|c|c|c|}
\hline & Suelo Control & Suelo contaminado & $\begin{array}{l}\text { Suelo contaminado con } \\
\text { tiosulfato de amonio } C_{1}\end{array}$ & $\begin{array}{l}\text { Suelo contaminado con } \\
\text { tiosulfato de amonio } \mathrm{C}_{2}\end{array}$ \\
\hline Total THg absorbido por planta $(\mu \mathrm{g})$ & & 3538.17 & 13931.98 & 16872.11 \\
\hline Mercurio extraído por $\mathrm{Kg}$ de suelo $(\mu \mathrm{g})$ & & 34000 & 41100 & 43700 \\
\hline BAF total & & 0.104 & 0.339 & 0.386 \\
\hline
\end{tabular}


Tabla 7. Cuadro comparativo con otras especies fitorremediadoras para mercurio

\begin{tabular}{|c|c|c|}
\hline Especie de planta & Acelerador químico & Efecto promotor \\
\hline Willow & $\mathrm{Kl}$ & $6.46-8.26 \mu \mathrm{g} / \mathrm{g}$ en plantas \\
\hline Poa annua & $\left(\mathrm{NH}_{4}\right)_{2} \mathrm{~S}_{2} \mathrm{O}_{3}$ & $2.66 \mathrm{mg} / \mathrm{kg}$ en raíces \\
\hline Brassica juncea & $\mathrm{NH}_{4} \mathrm{SCN}$ & $0.1 \mathrm{mg} / \mathrm{kg}$ (raíces), $0.1 \mathrm{mg} / \mathrm{kg}$ (hojas) \\
\hline Solanum nigrum $L$. & Biocarbón & $0.01,0.05,0.09$ y $0.1 \mathrm{mg} / \mathrm{kg}$ (acumulación de $\mathrm{Hg}$ en raíces después de 4, 5, 6 y 7 meses) \\
\hline Oryza sativa L. & Sulfuro fertilizante & 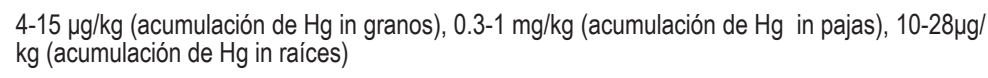 \\
\hline Helianthus annuus & $\left(\mathrm{NH}_{4}\right)_{2} \mathrm{~S}_{2} \mathrm{O}_{3}$ & $4.66 \mathrm{mg} / \mathrm{kg}$ (acumulación de $\mathrm{Hg}$ in hojas, $96.39 \mathrm{mg} / \mathrm{kg}$ ( acumulación de Hg in raíces) \\
\hline Brassica juncea & $\left(\mathrm{NH}_{4}\right)_{2} \mathrm{~S}_{2} \mathrm{O}_{3}$ & $71.5 \mathrm{mg} / \mathrm{kg}$ (acumulación de $\mathrm{Hg}$ en raices in roots), $41.5 \mathrm{mg} / \mathrm{kg}$ ( $\mathrm{Hg}$ accumulation en semillas) \\
\hline Festuca Dolichopylla* & $\left(\mathrm{NH}_{4}\right)_{2} \mathrm{~S}_{2} \mathrm{O}_{3}$ & $16.87 \mathrm{mg} / \mathrm{Kg}$ total en hojas. \\
\hline
\end{tabular}

Fuente: Liu et al. (2020) (*) Resultado del presente trabajo de investigación.

del suelo del distrito de Ananea, reduciendo efectivamente la cantidad de mercurio en los depósitos de metal de este suelo y, por lo tanto, podría ser utilizado para reducir el riesgo medioambiental en un plazo aceptable. Sin embargo, cualquier operación en la que se apliquen productos químicos al suelo para promover la solubilidad de los metales debe realizarse y manejarse con cuidado.

\section{CONCLUSIONES}

Los datos presentados en este trabajo sugieren que el tiosulfato de amonio puede aumentar la solubilidad de THg y, por lo tanto, mejorar la absorción de mercurio por la Festuca Dolichopylla. Esto se logra mediante la adición de tiosulfato a razón de $2 \mathrm{~g}$ de químico por kg de suelo, cinco días antes de la cosecha de las plantas. La mayor acumulación se da en las hojas, seguido por la raíz y el tallo.

El BAF hallado para la Festuca Dolichopylla es de 0.38 por lo que se concluye que no es una planta hiperacumuladora para mercurio.

El contenido de $\mathrm{THg}$ se redujo en el suelo al final del experimento en relación en el suelo inicial, en $43.67 \mathrm{mg} / \mathrm{kg}$, obteniéndose una eficacia de hasta $82 \%$.

Teniendo en cuenta los resultados, la fitorremediación de mercurio con la Festuca Dolichopylla asistida por tiosulfato puede usarse para manejar el nivel de contaminación de mercurio del suelo del distrito de Ananea, el cual reduciría efectivamente la cantidad de mercurio en los suelos.

\section{AGRADECIMIENTOS}

A las autoridades, docentes y estudiantes de la carrera profesional de Ingeniería Ambiental y Forestal de la Universidad Nacional de Juliaca por facilitar y apoyar el desarrollo del trabajo de investigación.

Al Vicerrectorado de Investigación y Post Grado de la Universidad Nacional Mayor de San Marcos, por el apoyo brindado al Proyecto con Código C19160013, sin financiamiento para la publicación del presente artículo.

\section{REFERENCIAS}

Arnau, J., \& Bono, R. (1997). Diseños experimentales: prácticas. Universitat de Barcelona. https://books.google.com.pe/ books?id=hDOwdygOVKUC\&lpg=PA1\&dq=Arnau $\% 2 \mathrm{C} \% 20$ J.\%2C\%20\%26\%20Bono\%2C\%20R.\%20(2017).\%20 Dise $\%$ C 3 \% B 1 os $\% 20$ Experimentales $\% 3$ A \% 20 Pr\%C3\%Alcticas.\%20Barcelona\%3A\%20Universitat $\% 20 \mathrm{de} \% 20$ Barcelona.\&lr\&hl $=$ es\&pg $=\mathrm{PP} 1 \mathrm{H} \mathrm{v}=$ onepage $\& \mathrm{q} \& \mathrm{f}=$ false

Chávez Rodríguez, L. (2014). Fitorremediacion con especies nativas en suelos contaminados por plomo [Universidad Agraria La Molina-UNALM]. In fitorremediacion con especies nativas en suelos contaminDOS POR PLOMO. https://repositorio.lamolina.edu.pe/handle/UNALM/2435

Dirección de Promoción Minera del Ministerio de Energía y Minas, \& (MINEM). (2020). Boletín Estadístico Minero 2020. http://www.minem.gob.pe/minem/archivos/file/Mineria/ PUBLICACIONES/VARIABLES/2020/BEMMAR20.pdf

Dorronsoro, C., \& García, I. (2005). Contaminación por metales. Departamento de Edafologia y Quimica Agricola. http:// edafologia.ugr.es/conta/tema15/introd.htm

García-Sánchez, A., Murciego, A., Álvarez-Ayuso, E., Regina, I. S., \& Rodríguez-González, M. A. (2009). Mercury in soils and plants in an abandoned cinnabar mining area (SW Spain). Journal of Hazardous Materials, 168(2-3), 13191324. https://doi.org/10.1016/j.jhazmat.2009.03.009

Geng, N., Wu, Y., Zhang, M., Tsang, D. C. W., Rinklebe, J., Xia, Y., Lu, D., Zhu, L., Palansooriya, K. N., Kim, K. H., \& Ok, Y. S. (2019). Bioaccumulation of potentially toxic elements by submerged plants and biofilms: A critical review. In Environment International (Vol. 131). https://doi. org/10.1016/j.envint.2019.105015

Liu, Z., Chen, B., Wang, L. ao, Urbanovich, O., Nagorskaya, L., Li, X., \& Tang, L. (2020). A review on phytoremediation of mercury contaminated soils. In Journal of Hazardous Materials (Vol. 400). https://doi.org/10.1016/j. jhazmat.2020.123138

Lomonte, C., Doronila, A. I., Gregory, D., Baker, A. J. M., \& Kolev, S. D. (2010). Phytotoxicity of biosolids and screening of selected plant species with potential for mercury phytoextraction. Journal of Hazardous Materials, 173(1-3), 494-501. https://doi.org/10.1016/j.jhazmat.2009.08.112 
Macera Poli, D. (2018). Logros y Retos del Desarrollo Económico Social de Puno. Foro Puno 2019. http://www.ipe.org. pe/portal/wp-content/uploads/2018/05/Foro-Puno-2018Logros-y-Retos-del-Desarrollo-Económico-y-Social-dePuno-Diego-Macera.pdf

Marrugo-Negrete, J., Durango-Hernández, J., Díaz-Fernández, L., Urango-Cárdenas, I., Araméndiz-Tatis, H., VergaraFlórez, V., Bravo, A. G., \& Díez, S. (2020). Transfer and bioaccumulation of mercury from soil in cowpea in gold mining sites. Chemosphere, 250. https://doi.org/10.1016/j. chemosphere.2020.126142

MINAM. (2014). Guia para el muestreo de suelos (R.M. 085-2014-MINAM) (Vol. 2, p. 91). http://www.minam. gob.pe/wp-content/uploads/2018/07/GUIA-PARA-ELMUESTREO-DE-SUELO.pdf

Ortiz, R., \& Aranibar, M. (2015). Plantas acumuladoras de metales en relaves mineros del altiplano de la región Puno. Investigación Andina, 15(2), 1-12. https://www.revistas. uancv.edu.pe/index.php/RCIA/article/view/36

SINIA. (2015). Dictan reglas para la presentación y evaluación del Informe de Identificación de Sitios Contaminados Designan Jefe del Servicio Nacional de Certificación Ambiental para las Inversiones Designan integrante del Consejo Directivo de PROFONANPE. Normas Legales, DECRETO SU, 565071-565072. www.senace.gob.pe
Teng, D., Mao, K., Ali, W., Xu, G., Huang, G., Niazi, N. K., Feng, X., \& Zhang, H. (2020). Describing the toxicity and sources and the remediation technologies for mercury-contaminated soil. In RSC Advances (Vol. 10, Issue 39, pp. 23221-23232). https://doi.org/10.1039/d0ra01507e

Wang, J., Anderson, C. W. N., Xing, Y., Fan, Y., Xia, J., Shaheen, S. M., Rinklebe, J., \& Feng, X. (2018). Thiosulphateinduced phytoextraction of mercury in Brassica juncea: Spectroscopic investigations to define a mechanism for $\mathrm{Hg}$ uptake. Environmental Pollution, 242, 986-993. https://doi. org/10.1016/j.envpol.2018.07.065

Wang, J., Feng, X., Anderson, C. W. N., Qiu, G., Ping, L., \& Bao, Z. (2011). Ammonium thiosulphate enhanced phytoextraction from mercury contaminated soil - Results from a greenhouse study. Journal of Hazardous Materials, 186(1), 119-127. https://doi.org/10.1016/j.jhazmat.2010.10.097 